\title{
Identification of Bacteria by Computer: General Aspects and Perspectives
}

\author{
By S. P. LAPAGE, SHOSHANA BASCOMB*, W. R. WILLCOX \\ AND M. A. CURTIS \\ Computer Trials Department, National Collection of Type Cultures, Central \\ Public Health Laboratory, Colindale Avenue, London, $\mathrm{NW}_{9}{ }_{5} \mathrm{HT}$
}

(Received 8 November 1972; revised 19 March 1973)

SUMMARY

The general problems of probabilistic identification of bacteria are discussed, particularly choice of taxa, choice of tests, assignation of probabilities and variation with geographical distribution, method of modification of probabilities as information is obtained, identification levels and linkage of tests.

A study is described of the probabilistic identification of 1079 reference strains, and 516 field strains of Gram-negative, aerobic, rod-shaped bacteria. The field strains were identified both on the test results obtained by sending laboratories and test results in our laboratory. Identification rates for fermentative bacteria of $90.8 \%$ for reference strains and $89.4 \%$ for field strains were obtained, and for non-fermentative bacteria of $82 . \mathrm{I} \%$ and $70.8 \%$ respectively. The field strains were received because they were difficult to identify in the medical diagnostic laboratory; higher rates of identification might be expected for typical strains.

\section{INTRODUCTION}

Many of the problems of microbial identification were discussed by Steel (I962, I965), but the use of numerical methods in identification has been comparatively neglected although they are widely used in classification.

Beers \& Lockhart (I962) suggested three possible lines of approach in numerical identification. The first method was to carry out a numerical classification for each new isolate. This would seem impracticable for single strains but might be used for batch processing of large numbers. Quadling \& Colwell (1964), Gyllenberg (I965) and J. C. Gower and G. J. S. Ross (personal communications) have described methods for identification by the comparison of the unidentified strains with previously constructed taxonomic groups using numerical clustering methods.

The second suggested method was to construct keys mathematically and use them to replace those used in conventional identification. Maccacaro (1958) had suggested that keys could be made by evaluation of the 'information content' of each test. Hill \& Silvestri (I962) constructed a probability key for the diagnosis of Actinomycetales and the mathematical basis for this key was described by Möller (1962). Gyllenberg (1963) used an estimation of differentiating power to choose tests, a method which was applied by Rypka, Clapper, Bowen \& Babb (1967) and further developed by Rypka \& Babb (I970). Niemelä, Hopkins \& Quadling (I968) also described a method for test selection based on information theory. In all these methods, a mathematical model is used once to determine the 'best'

* Present address: Department of Biochemistry, Imperial College, Imperial Institute Road, London, SW7 2AZ. 
tests, and strains are then identified by comparison of their test results with those expected for each taxon by conventional methods. Although the advantage of a reduction in the number of tests required for identification can be expected, these methods have the same disadvantage as conventional (intuitive) identification - the difficulty of identifying strains aberrant in one or more characters.

The third approach suggested by Beers \& Lockhart (1962) was to estimate the probability of strains of each taxon giving positive results in each test and then to use these probabilities to yield a statistical estimate of the reliability of the diagnosis. Payne (I963) adopted this method, working with Dybowski \& Franklin (1968), who gave a detailed account of a conditional probability method and its application to the identification of enterobacteria. Lapage, Bascomb, Willcox \& Curtis (1970) described the use of a similar probability method in the identification of Gram-negative rod-shaped bacteria. A method was also described for the selection of tests with the highest discriminating power between the possible taxa suggested as likely identifications by the probability computations. Sneath (1969) discussed the theoretical effect which test differences would have on identification.

Dito (I97I) has devised an economical system for the storage of strain data. The results found on unknown strains can be matched with those stored for previously identified strains and the unknown strains thus identified or the occurrence of a new pattern of results signalled. Operation of the system on an electronic desk calculator is also described. A similar approach was used by Fey (1959) and Baer \& Washington (1972).

\section{PRESENT INVESTIGATION}

A probability method was investigated in the present study. The statistical method was based on Bayes's theorem but except for a few cases could equally be based on relative likelihood (Willcox, Lapage, Bascomb \& Curtis, 1973).

Gram-negative, rod-shaped bacteria were chosen as a model and a table (matrix) was constructed which contained the estimated probabilities of a positive result in each test for each taxon. The results of tests on an unknown strain were compared with the stored probabilities and the likelihood that the strain belonged to each of the matrix taxa was calculated in turn by multiplication of the relevant probabilities. These likelihoods were normalized and the value for each taxon referred to as the identification score for that taxon. This procedure was applied to reference strains and to freshly isolated strains (field strains) from various medical diagnostic bacteriological laboratories (chiefly of the Public Health Laboratory Service). The resulting data were used to improve the matrix, investigate the number of tests needed for identification, estimate test differences between laboratories and for test selection strategies. The matrix was stored in a computer in which all calculations were carried out.

In the following three papers we describe in detail the methods used from 1967 to 1970 for the identification of 1595 bacterial strains and the problems arising from the application of these methods. The first paper deals generally with problems of such a project, the second gives the bacteriological account, and the third discusses the mathematical aspects.

\section{METHODS}

Choice of group of bacteria. Gram-negative, rod-shaped bacteria which grow aerobically on nutrient agar at 37 or $22{ }^{\circ} \mathrm{C}$ were chosen, e.g. enterobacteria, pseudomonads and some parvobacteria (Bascomb, Lapage, Curtis \& Willcox, I973). This group was selected because 
it has been extensively studied and many previously identified strains were available for reference purposes. Moreover, these bacteria are easy to handle and test and survive well in the laboratory.

\section{Strains}

Reference strains. A total of 1028 reference strains were examined afresh in all the $5 \mathrm{I}$ tests in the matrix (Bascomb et al. 1973). These strains, chiefly from culture collections and similar sources, were used to complete and test the matrix probabilities and additional confirmation of their identities was provided by tests not included in the matrix, e.g. serological or further biochemical tests or tests of bacteriophage sensitivity. The identity of each of these cultures was first confirmed by conventional methods from the available results of all the tests by at least two of us, and the strains were then identified by the computer method (identification by intuitive assessment of the test results is referred to as conventional identification, and by computation as computer identification). The results of the two methods were compared and any reference strain which could not be identified by either of these means, or which did not agree in its characters with the name supplied by the original source, was excluded from this study.

Strains of taxa not in the matrix. A total of 5I strains which belonged to taxa not included in the matrix were tested in the same way as the reference strains and then identified by the computer method.

Field strains. A total of 516 field strains were identified both by the limited number of results provided by the sending laboratories and by the results of the complete set of tests in our laboratory. The operation of this system was described in Lapage et al. (1970).

\section{Construction of a matrix.}

Choice of taxa. Conventional bacterial taxa were used (Bascomb et al. 1973) and a matrix constructed for taxa containing pathogenic species, taxa known to be found in medical specimens, and a number of others. An exhaustive matrix of all Gram-negative aerobic or facultatively anaerobic species was not practicable nor were the data available, although such taxa might be found by chance in medical specimens. The taxa in the matrix were not of equal taxonomic rank since some have been studied in greater detail, and because strains of some taxa show a wider variation in their properties. Well-defined homogeneous taxa, e.g. Salmonella typhi, were included while others contained strains with a greater range of variation, e.g. Escherichia coli, and some were broad groups, e.g. Moraxella spp. Hence they are referred to throughout this account as taxa rather than species.

Choice of tests. Tests were chosen which were reproducible and easy to prepare, carry out and read. The range chosen (Table I) included those commonly used in the medical diagnostic bacteriological laboratory but subjective tests such as colonial morphology were avoided. A range of tests was selected which included tests for as many different enzymes and biochemical pathways as possible, e.g. the electron transport system and those involved in the metabolism of carbon and nitrogen compounds.

Most of the tests were qualitative and could be expressed in two states. The use of quantitative tests, split into a set of qualitative steps, is not precluded in our program and techniques suitable for multistate characters were used for the various hues of pigment.

To hasten results and avoid the lack of reproducibility inherent in late and irregular positive results, all the tests were read at I, 2 and finally at 5 days, except gelatin liquefaction which was then read up to 28 days. Details of the media and methods, and of the methods of scoring are given in Bascomb, Lapage, Willcox \& Curtis (1971). The independence of the 
Table I. Analysis of 13029 tests

Urease, presence

Indole production

Lactose, acid production

Glucose, acid production

Mannitol, acid production

Gas production from glucose

Sucrose, acid production

MacConkey agar, growth

Maltose, acid production

Oxidase, presence

Growth at $37^{\circ} \mathrm{C}$

Dulcitol, acid production

Salicin, acid production

Simmons' citrate, growth

$\mathrm{H}_{2} \mathrm{~S}$ production (lead acetate paper)

Gelatin, liquefaction within 5 days

$\mathrm{KCN}$ medium, growth

Catalase, presence

Gluconate reduction

Malonate utilization

Pigment production

Motility at $37^{\circ} \mathrm{C}$

Hugh and Leifson test

oxidative

fermentative

alkali production

Deamination of phenylalanine (PPA)

Arginine, dihydrolase

Ornithine, decarboxylase

Lysine, decarboxylase

Inositol, acid production

Adonitol, acid production

ONPG ( $\beta$-galactosidase)

Growth at RT

Methyl red at RT

Nitrate reduction

Arabinose, acid production

Motility at RT

Voges-Proskauer at RT

Xylose, acid production

$\mathrm{H}_{2} \mathrm{~S}$ production (TSI)

Sorbitol, acid production

Voges-Proskauer at $37^{\circ} \mathrm{C}$

Methyl red at $37^{\circ} \mathrm{C}$

Trehalose, acid production

Gelatin, liquefaction within 28 days

$\begin{array}{ccc}\text { Frequency of use } & \text { Separation value } & \begin{array}{c}\text { Interlaboratory } \\ \text { test differences }\end{array} \\ \text { by sender }(\%) & (\text { maximum }=1225) & (\%)\end{array}$

Glycerol, acid production

Raffinose, acid production

Rhamnose, acid production

Cellobiose, acid production

Starch, acid production

Selenite reduction

\begin{tabular}{|c|c|}
\hline 95 & 528 \\
\hline 95 & 816 \\
\hline 94 & 624 \\
\hline 88 & 522 \\
\hline 82 & 1080 \\
\hline 82 & 984 \\
\hline $8 \mathrm{I}$ & 884 \\
\hline 80 & 216 \\
\hline 79 & 945 \\
\hline 75 & 736 \\
\hline 75 & 134 \\
\hline 74 & 399 \\
\hline 72 & 630 \\
\hline 68 & 736 \\
\hline 67 & 516 \\
\hline 59 & 689 \\
\hline 57 & 910 \\
\hline 54 & 8 \\
\hline $5 I$ & 513 \\
\hline $5 \mathrm{I}$ & 650 \\
\hline 50 & 118 \\
\hline 48 & 899 \\
\hline 47 & - \\
\hline$\longrightarrow$ & 434 \\
\hline- & 686 \\
\hline- & 256 \\
\hline 45 & 384 \\
\hline 42 & 860 \\
\hline $4 \mathrm{I}$ & 988 \\
\hline $4 \mathrm{I}$ & 840 \\
\hline 39 & 636 \\
\hline 39 & 495 \\
\hline 39 & 1054 \\
\hline 37 & 236 \\
\hline 36 & 1020 \\
\hline 36 & 248 \\
\hline 35 & I I 56 \\
\hline 32 & 1092 \\
\hline 32 & 406 \\
\hline 25 & 870 \\
\hline 25 & 627 \\
\hline 23 & 975 \\
\hline 19 & 180 \\
\hline 18 & $9 \mathrm{I} 8$ \\
\hline 17 & 924 \\
\hline 16 & 760 \\
\hline I5 & 64 \\
\hline 12 & 728 \\
\hline I I & \\
\hline 10 & . \\
\hline 8 & \\
\hline 0.2 & \\
\hline
\end{tabular}

TSI, triple sugar iron medium.

$\mathrm{RT}$, room temperature or incubator at 22 or $30^{\circ} \mathrm{C}$.

* Includes tests of both fermentative and non-fermentative bacteria.

$\uparrow$ The selenite test was only carried out by the sending laboratories on a few strains. 
tests used and the treatment of linked tests are discussed below and the mathematical treatment described in Willcox et al. (I973).

\section{Assignation of probabilities for the stored matrix}

Preliminary matrix. This was constructed semiquantitatively $(+, \pm, d, \mp,-)$ for 90 taxa in 70 tests from published data, from records of the N.C.T.C. and of other laboratories at the Central Public Health Laboratory, Colindale. It was difficult to compile a satisfactory matrix due to lack of data, particularly of data derived from standardized methods of testing. Bacteriological papers are not usually written for the display of detailed strain data nor would editors accept them as such. The population under sample is often ill-defined and different methods of scoring such as 'delayed' or 'variable' prove difficult to interpret as are phrases such as 'few', 'many' or 'some'. Further, the distribution of results from different geographical areas may vary. The problem is then whether to include several biotypes in the matrix, in which case it might turn out to be impossible to discriminate between these biotypes by the probabilistic identification method, or whether to use a single taxon with several 'variable' matrix entries in which case it might be difficult to separate this taxon from other taxa in the matrix. Similarly, a taxon which is too inclusive and contains subgroups may need division, e.g. Citrobacter was divided in the present matrix (Bascomb et al. 1973). Therefore many decisions of expediency were made for this preliminary matrix.

Matrix 66. From the preliminary matrix and from the results of tests on reference strains, a first quantitative matrix (Matrix 66) was constructed which contained 62 taxa and 50 tests; 28 taxa and 20 tests were deleted from the preliminary matrix because there was inadequate information, or because the tests had little overall differentiation between the taxa. The probabilities assigned in Matrix 66 to the qualitative values of the preliminary matrix are shown in Table 2.

When all the strains of a taxon were known to give a positive result or known exceptions were very rare, a value of 0.99 was allotted and a corresponding value of $0.0 \mathrm{I}$ when all were negative. The relevant matrix entry gives the probability of a positive result and one minus the entry the probability of a negative result. The justification for the use of these probabilities is given in Willcox et al. (1973). However, by this quantification of the preliminary matrix, the distinction was lost between an event considered impossible or extremely improbable, which could have been allotted a probability of 0.999 or higher, and an event less improbable or known to occur very rarely, given perhaps a probability of 0.99. In some systems, a range of scores to represent different degrees of certainty might be valuable and data from large samples available. However, to produce accurate figures for bacteria with reasonable statistical confidence, thousands of strains of bacteria must be tested by standardized methods which is not at the moment, for us, a practicable proposition.

When the strains were mostly positive but exceptions were found a value of 0.95 was allotted in Matrix 66, and 0.05 when the strains were largely negative (Table 2). When exceptions were known to be common, values of 0.85 and 0.15 were given. Other measured probabilities derived either from tests on a number of strains, or from published data, were recorded at 0.05 intervals, e.g. $0.65,0.30$, as the available data and accuracy of the method did not justify finer subdivisions.

The decision to use values of 0.99 and $0.0 \mathrm{I}$ as the upper and lower limits of probability was justified by the assumption of an error rate of $\mathrm{I} \%$ for any result. The sources of error are manifold and we have found in this study that between laboratories an overall test result difference rate of $7.9 \%$ can be expected which, using the formulae of Sneath \& Johnson (I972), represents an error rate of $4 . \mathrm{I} \%$. Past experience would lead us to expect that some, 
Table 2. Methods of scoring in matrices

Basis of matrix entry

\begin{tabular}{|c|c|c|c|}
\hline \multirow[b]{2}{*}{ Concept } & \multirow{2}{*}{$\begin{array}{l}\text { Percentage of } \\
\text { strains found } \\
\text { positive (if } \\
\text { available) }\end{array}$} & \multicolumn{2}{|r|}{ Entry made } \\
\hline & & $\begin{array}{l}\text { Pre- } \\
\text { liminary } \\
\text { matrix }\end{array}$ & $\begin{array}{l}\text { Matrix } 66 \\
\text { and } \\
\text { Matrix } 68\end{array}$ \\
\hline Negative results not known and hard to conceive & 100 & & \\
\hline Negative results conceivable but if found extremely rare & 99 or above & + & \\
\hline Negative results found but not common & $9 I-98$ & & 0.95 \\
\hline Usually positive but exceptions common & $8 I-90$ & \pm & 0.85 or 0.9 \\
\hline Distribution of positive results & $20-80$ & $\mathrm{~d}$ & $0.2-0.8$ in steps of 0.05 \\
\hline Usually negative but exceptions common & $10-19$ & $\mp$ & 0.1 or 0.15 \\
\hline Positive results found but not common & $2-9$ & ) & 0.05 \\
\hline $\begin{array}{l}\text { Positive results conceivable but if found extremely rare } \\
\text { Positive results not known and hard to conceive }\end{array}$ & $\begin{array}{l}\text { I or below } \\
\text { o }\end{array}$ & - & \\
\hline
\end{tabular}

though not as great an error rate, would occur in repetition in the same laboratory. In their series of tests Sneath \& Johnson (1972) found an error rate of $2 \cdot 2 \%$ from carefully performed tests in one laboratory. Snell \& Lapage (1973) found a rate of $5 \%$ for carbon source utilization tests repeated in the laboratory. Also, in any combined clerical operation a rate of up to I $\%$ can perhaps be expected due to misreading, misscoring and clerical errors, in addition to the laboratory error rate due to contaminated cultures and faulty media. Finally, a probability of error of $0.0 \mathrm{I}$ is less than the 0.05 steps used for the data in the matrix $(0.04$ in the special cases of $0.99-0.95$ and $0.05-0.01$ ). The application of the data error method is described by Willcox et al. (I973) and enabled us to set limits to the probabilities and provide statistical justification of our method.

Matrix 68. After further reference and field strains had been tested, and experimental matrices tried for particular taxa in Matrix 66, an improved second quantitative matrix (Matrix 68), based on Matrix 66, was made with eight additional taxa and one additional test, thus comprising 70 taxa and 5I tests. The values for the tests allotted to the taxa in the matrix are presented in Bascomb et al. (1973). This matrix has been in use, with a few additions and some minor changes in the probability figures, since that time.

Uncompleted squares. Probabilities could not always be allotted for a given taxon in a given test for one of three reasons: the information was unavailable; the test was inapplicable under the standardized conditions of testing, e.g. production of a purple colour in the oxidase test if the bacterial strain produced a violet pigment, as with Chromobacterium violaceum (usually a result for the test could be obtained but the theoretical category still exists); or the test was meaningless since it could not be carried out in practice, e.g. a test for a property at a given temperature at which the strain did not grow. The method for dealing with uncompleted squares is described below and in Willcox et al. (1973).

Modifications to the matrix. The matrix was kept under continuous review and modified when new data became available, chiefly from tests on a larger number of reference strains by standardized methods in our laboratory. The results obtained from field strains also provided information particularly as these were samples of the population likely to be received for identification. Information from new published work was also considered and the results of any taxonomic studies that we had carried out. Tests introduced to our laboratory and new tests which we developed were added, and so were newly described taxa of bacteria. Experimental matrices were made for certain taxa in which identification proved difficult, e.g. Pasteurella, Shigella, and the arrangement found to identify the maximum 
Table 3. Examples of calculations of identification procedure

(I) Matrix

Taxa $\left\{\begin{array}{l}X \\ Y \\ Z\end{array}\right.$

Results of strain to be identified

\begin{tabular}{cccc}
\multicolumn{1}{c}{ Tests } & $c$ & $d$ \\
$a$ & $b$ & 0.01 & 0.01 \\
0.99 & 0.99 & 0.01 & 0.99 \\
0.99 & 0.80 & 0.99 & 0.99 \\
0.90 & 0.01 & & 0.99
\end{tabular}

Not done

(2) Calculations used in the present study

(a) Likelihoods for given test results

$$
\operatorname{Taxa}\left\{\begin{array}{rl}
X & 0.99 \times 0.99 \times(1-0.01)=0.970299 \\
Y & 0.99 \times 0.80 \times(\mathrm{I}-0.0 \mathrm{I})=0.784080 \\
Z & 0.90 \times 0.01 \times(\mathrm{I}-0.99)=0.000090 \\
\text { Sum } & =\mathrm{I} .754469
\end{array}\right.
$$

(b) Identification scores (normalized)

$$
\begin{aligned}
& \frac{0.970299}{1 \cdot 754469}=0.553044 \\
& \frac{0.784080}{1 \cdot 754469}=0.446905 \\
& \frac{0.000090}{1 \cdot 754469}=0.00005 \mathrm{I}
\end{aligned}
$$

(3) Calculations used by Dybowski \& Franklin (1968), following stage $2 a$

$$
\begin{aligned}
& \operatorname{Taxa} \begin{cases}X & \begin{array}{l}
\frac{0.970299}{0.970299}=100.0000 \% \\
Y
\end{array} \frac{0.784080}{0.970299}=80.8080 \% \\
Z & \frac{0.000090}{0.970299}=0.0093 \%\end{cases} \\
& \overbrace{\frac{0.970299}{0.970299}}=\mathrm{I} \cdot 000000 \\
& \frac{0.784080}{0.784080}=\mathrm{I} \cdot 000000 \\
& \frac{0.000090}{0.882090}=0.000102
\end{aligned}
$$

number of strains was incorporated into the main matrix after it had been shown to operate successfully within the extended framework.

All changes to the probabilities in the matrix were made by decision, and the computer program did not provide for automatic alteration on the results of each strain identified since it was not desirable that identification of a strain belonging to a taxon not in the matrix or an intermediate strain should alter the matrix probabilities automatically. In addition, since some of the taxa contained more than one species, e.g. Shigella, the reactions of the common local strain, say Shigella flexneri 2 a, might come to replace the probabilities in the matrix which allowed for the test results of the other serotypes and hence for their recognition. Such a swamping effect by strains of common local subsets of the taxon might create real difficulties in the identification of rarer subsets if a self-adjusting system was used. Finally, contaminated cultures, incorrect test results or errors in transcription might cause incorrect changes in the probabilities if they were automatically adjusted for each strain.

\section{Identification and test selection}

Calculation of likelihoods and identification scores. The calculation of the identification scores (Table 3) is based on Bayes's theorem (Willcox et al. 1973). The likelihood that the unknown strain is a member of a given taxon is defined as the probability that a member of this taxon will give the same pattern of test results as that observed on the unknown strain. The likelihood is calculated for each taxon in turn by multiplying together the probabilities, 


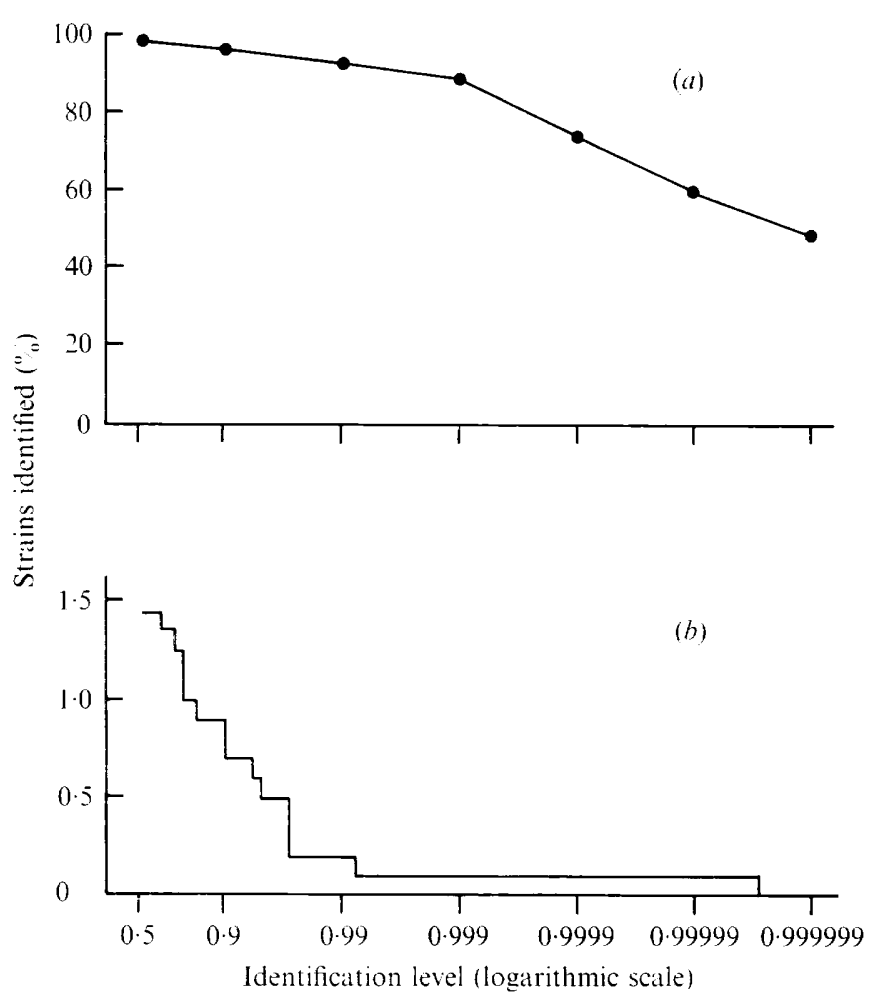

Fig. I. Effect of changing the identification level on the computer identification of r 028 reference strains. (a) Percentage of strains identified to correct taxon. (b) Percentage of strains identified to incorrect taxon. Because of the low number of strains involved $(b)$ is drawn as a stepped graph rather than as a smoothed curve between sampling points.

given that taxon, of each of the individual test results. These likelihoods are normalized by dividing each likelihood by the sum of the likelihoods for all the taxa. The normalized likelihoods are referred to as identification scores. Included in the basic method are allowances for logically linked and causally related tests, and for elements of the matrix whose value is unknown, all of which are discussed below and more fully in Willcox et al. (1973). The prior probabilities for all the taxa were treated as equal (Willcox et al. 1973) so that the possibility of the strain belonging to any of the taxa in the matrix was the same, irrespective of the rarity of the taxa, i.e. the strain was evaluated on its test results alone.

Identification level. Unprocessed likelihoods are difficult to evaluate. Dybowski \& Franklin (I968) expressed the likelihoods as proportions of the highest likelihood and called these values 'percentage relative likelihoods' (Table 3 ). Such a step enables the taxa to be ranked in order of likelihood, but does not provide a straightforward means for the determination of identification, as it is possible that two taxa will have the same high likelihood and both would therefore show a $100 \%$ score.

Normalization of likelihoods is inherent in the application of Bayes's theorem and the resulting scores can be compared with a threshold identification level. If the score of any taxon exceeds this level the strain is identified as a member of that taxon; if not, the strain is not identified. Thus normalized scores provide an unequivocal display by which identification can be immediately recognised unlike unprocessed likelihoods. A study of different identification levels showed that the level of identification score of 0.999 was the most 
favourable (Fig. I). Levels above were too infrequently obtained while levels below led to incorrect identifications. Since the scores are normalized, the sum of the scores of the other taxa must be less than 0.00I. The level of 0.999 represents a theoretical error rate of I in 1000 .

A computer identification printout was produced for each strain (Lapage et al. 1970). The printout included reference data on the strain, a list of tests carried out and the results obtained. If the strain was identified then the probability figures in the matrix for the chosen taxon in each test were printed.

The use of normalized probabilities creates at least three problems. Firstly, a high identification score is possible if the probability product for one taxon is much higher than that for any other. Such a situation may occur with any level of probability product and actually happened in one case where the likelihood of the highest taxon was less than $\mathrm{I} \times \mathrm{IO}^{-12}$. This problem was discussed by Dybowski \& Franklin (I968), who attempted to circumvent it by devising the modal likelihood fraction. By this device the actual likelihood for the unknown strain in each taxon is divided by the maximum possible likelihood for a strain of that taxon as shown in Table 3. Although such a strain might achieve the top score in their system, it would show a low fraction of the modal likelihood. We preferred to print out the tests in which the strain differed from the matrix when the strain was + or - and the matrix figures 0.0 I or 0.99 respectively. It should be possible to put a maximum level of such test differences above which identification is unacceptable. Such a situation is likely to occur with strains of taxa not included in the matrix.

The second problem results from the difference in homogeneity of taxa in the matrix. A taxon whose strains are more homogeneous, i.e. with more values of 0.99 or 0.01 in the matrix for the relevant tests, will have a higher identification score than a less homogeneous taxon in the same tests since the probabilities for the given results in these tests are more likely to be below 0.99 or above 0.0 I (see taxa $X$ and $Y$ in Table 3). Dybowski and Franklin's modal likelihood fraction would minimize such differences. However, without further evidence about such a strain, it is statistically more likely to belong to the more homogeneous taxon and the answer is to carry out further tests.

The third problem again depends on the matrix figures. Although it cannot be demonstrated strictly that at least two 0.99 or 0.0 I differences were required for the separation of two taxa, a favourable identification rate cannot be assumed unless at least two such differences are found. However, an identification rate of $100 \%$ may be achieved even when two taxa are separated by one or even no 0.99/0.0 differences (Bascomb et al. 1973). If the matrix contains two taxa similar in most results and artificially separated taxonomically but with a higher identification rate if kept separate, e.g. Escherichia coli and the Alkalescens-Dispar group, then the likelihood of a strain belonging to either of these taxa will be high for both, and the identification score of each will approach 0.5 . In this case an identification score for a taxon was accepted if the sum of the scores of the two specified taxa exceeded 0.999, and identification was considered to be in a taxon comprising the two constituent taxa.

Uncompleted squares. An uncompleted square was regarded as containing no information so the square was first set to agreement with the test result, e.g. 0.99 if the strain gave a positive result, and the likelihood calculated for that taxon. The process was carried out for each taxon in which there was an uncompleted square for the given tests. Then, for the highest scoring taxon only, the square was set to disagreement, e.g. o.o I for a positive result, and the score was recalculated (Willcox et al. I973). If the strain identified in the same taxon on both scores then the identification was accepted. If it identified on the highest but not the lowest, the position was assessed. If it had failed to identify on the highest score, calculation of the lowest score was not considered necessary. In practice this method could 
be used as uncompleted squares were few. If more than one square was uncompleted for a given taxon then the highest and lowest scores only would be required for a given taxon and these were calculated by setting all the squares concerned to agreement or disagreement. This method was used for 'logical' and 'inapplicable' types of linked test. In the present study, all the uncompleted squares of the 'meaningless' type were due to inability to test a property, e.g. motility, because a particular strain did not grow at the given temperature, e.g. $37{ }^{\circ} \mathrm{C}$, and since the test for growth at this temperature was also in the matrix such uncompleted squares were treated as linked tests (see below).

Linked tests. Tests may be linked for three reasons. (i) Causal: the same properties are tested by different methods, e.g. $\beta$-galactosidase production (ONPG test) and lactose fermentation, although they may only be linked in particular species since a positive result may be given in the same test by different bacteria but by different biochemical pathways e.g. acid production from lactose by breakdown and metabolism of the products, or by formation of lactobionic acid. (ii) Logical: when by definition a positive result in one test implies a positive (or negative) result in another, e.g. if gelatin liquefaction is scored as two tests, liquefaction in I to 5 and I to 28 days, then if gelatin was liquefied in $I$ to 5 days it must be liquefied within I to 28 days. (iii) Statistical: when, with adequate samples, estimates can be made for each taxon of the probability of the test result in a given test in relation to the probabilities of the test results in the other tests.

For linked tests the probabilities of the individual test results cannot simply be multiplied together but the particular combination of test results observed must be considered. When two linked tests are both positive (or negative) one is ignored as one result implies the other. By definition one of the linked tests cannot be positive when the other is negative, e.g. motility at a temperature at which the organism does not grow, and the combination is signalled as illegitimate by the computer. When the permitted combination of positive and negative is found the calculation is complex and the probability is estimated from the sum of the probabilities of the other possible situations subtracted from I 0 (see Willcox et al. 1973, for further details).

In Matrix 66 the initial uncompleted squares necessitated the use of the method of rescoring uncompleted squares and inapplicable tests, as well as the method for linked tests. In Matrix 68 all the squares had been completed so rescores were only used for meaningless tests. The linked test methods were used, and the data error method used throughout.

Test selection. If the strain does not identify on the basis of results from some of the tests included in the matrix then the identification scores of the taxa are added together in order of rank, starting with the highest, until the sum of their scores is equal to, or exceeds, 0.999. The group of taxa so formed are considered to be possible identifications and the matrix entries of these taxa for the tests not so far performed are then reduced to $a+,-$ and $o$ form, entries $\geqslant 0.85$ being set to,$+ \leqslant 0.15$ to - , and the remainder to 0 .

The value of the tests, thus reduced, in separating the taxa was calculated using the separation value (Gyllenberg, 1963) which is the product of the number of taxa positive in the test and the number negative. The separation value of a test represents the number of pairs of taxa which the test will separate. The separation values for the tests in the present investigation are given in Table I. The first column shows the frequency of use of the tests by the sending laboratories, the second the separation value, and the third the percentage of test result differences between the sending and our laboratory.

Tests are selected sequentially according to the number of pairs of the possible taxa which they separate. A test separates a pair of taxa if it is + in one taxon and - in the other. 
Table 4. Identification of fermentative bacteria

\begin{tabular}{|c|c|c|c|c|c|c|c|c|}
\hline & \multirow{3}{*}{$\begin{array}{c}\text { Number } \\
\text { of } \\
\text { strains }\end{array}$} & \multirow{2}{*}{\multicolumn{2}{|c|}{$\begin{array}{l}\text { Percentage of strains } \\
\text { identified }\end{array}$}} & \multicolumn{5}{|c|}{$\begin{array}{c}\text { Comparison of conventional and computer } \\
\text { identifications }(\%)\end{array}$} \\
\hline & & & & & & Conven- & Com- & \\
\hline & & $\begin{array}{l}\text { Conven- } \\
\text { tionally }\end{array}$ & $\begin{array}{c}\text { By } \\
\text { computer }\end{array}$ & $\begin{array}{l}\text { Same } \\
\text { taxon }\end{array}$ & $\begin{array}{l}\text { Different } \\
\text { taxa }\end{array}$ & $\begin{array}{l}\text { tional } \\
\text { only }\end{array}$ & $\begin{array}{l}\text { puter } \\
\text { only }\end{array}$ & $\begin{array}{l}\text { Identified } \\
\text { by neither }\end{array}$ \\
\hline \multicolumn{9}{|l|}{ Reference strains } \\
\hline Matrix 66 & 686 & 100 & $8 I \cdot 3$ & $80 \cdot 3$ & $I \cdot O$ & $18 \cdot 7$ & 0 & 0 \\
\hline Matrix 68 & 827 & 100 & $90 \cdot 9$ & $90 \cdot 8$ & $0 \cdot I$ & $9 \cdot I$ & 0 & 0 \\
\hline \multicolumn{9}{|l|}{ Field strains } \\
\hline $\begin{array}{l}\text { Matrix } 66, \text { limited no. } \\
\text { of tests* }\end{array}$ & 226 & $87 \cdot 6$ & $79 \cdot 6$ & $74 \cdot 3$ & $2 \cdot 2$ & $I I \cdot I$ & $3 \cdot I$ & $9 \cdot 3$ \\
\hline Matrix 66 , all tests & 226 & $87 \cdot 6$ & $8 I \cdot 9$ & $77 \cdot 0$ & $\mathrm{I} \cdot 8$ & $8 \cdot 8$ & $3 \cdot I$ & $9 \cdot 3$ \\
\hline Matrix 68 , all tests & 359 & $88 \cdot 8$ & $84 \cdot 4$ & $8 I \cdot 3$ & 0 & $7 \cdot 5$ & $3 \cdot I$ & $8 \cdot \mathrm{I}$ \\
\hline
\end{tabular}

Table 5. Identification of non-fermentative bacteria

\begin{tabular}{|c|c|c|c|c|c|c|c|c|}
\hline & \multirow{3}{*}{$\begin{array}{c}\text { Number } \\
\text { of } \\
\text { strains }\end{array}$} & \multirow{2}{*}{\multicolumn{2}{|c|}{$\begin{array}{l}\text { Percentage of strains } \\
\text { identified }\end{array}$}} & \multicolumn{5}{|c|}{$\begin{array}{c}\text { Comparison of conventional and computer } \\
\text { identifications }(\%)\end{array}$} \\
\hline & & & & & & Conven- & Com- & \\
\hline & & $\begin{array}{l}\text { Conven- } \\
\text { tionally }\end{array}$ & $\begin{array}{c}\text { By } \\
\text { computer }\end{array}$ & $\begin{array}{l}\text { Same } \\
\text { taxon }\end{array}$ & $\begin{array}{l}\text { Different } \\
\text { taxa }\end{array}$ & $\begin{array}{c}\text { tional } \\
\text { only }\end{array}$ & $\begin{array}{l}\text { puter } \\
\text { only }\end{array}$ & $\begin{array}{l}\text { Identified } \\
\text { by neither }\end{array}$ \\
\hline \multicolumn{9}{|l|}{ Reference strains } \\
\hline Matrix 66 & I34 & 100 & $43 \cdot 3$ & $41 \cdot 8$ & $I \cdot 5$ & $56 \cdot 7$ & 0 & 0 \\
\hline Matrix 68 & 201 & 100 & $82 \cdot 1$ & $82 \cdot I$ & 0 & $17 \cdot 9$ & 0 & 0 \\
\hline \multicolumn{9}{|l|}{ Field strains } \\
\hline $\begin{array}{l}\text { Matrix } 66, \text { limited } \\
\text { no. of tests* }\end{array}$ & 49 & $75 \cdot 5$ & $44 \cdot 9$ & $36 \cdot 8$ & $2 \cdot 0$ & $36 \cdot 7$ & $6 \cdot 1$ & $18 \cdot 4$ \\
\hline Matrix 66 , all tests & 49 & $75 \cdot 5$ & $42 \cdot 9$ & $38 \cdot 8$ & 0 & $36 \cdot 7$ & $4 \cdot 1$ & 20.4 \\
\hline Matrix 68 , all tests & I 57 & $65 \cdot 6$ & $52 \cdot 2$ & $44^{\circ} 0$ & 0.6 & $2 I \cdot 0$ & $7 \cdot 6$ & $26 \cdot 8$ \\
\hline
\end{tabular}

When a pair of taxa is separated by two tests it is considered to be completely separated. At each stage that test is chosen which separates the maximum number of those pairs of taxa which have not been completely separated by tests already selected (Lapage et al. 1970; Willcox et al. 1973).

\section{RESULTS}

Results for the reference and field strains are shown in Tables 4 and 5, arranged by fermentative and non-fermentative taxa respectively. The reference strains were all examined on both Matrix 66 and 68. All 516 field strains were examined on Matrix 68 using the results of all the tests in the matrix, but only 275 field strains were examined by both all and the limited set of tests on Matrix 66. The limited number of tests were those which resulted from the sending laboratories' initial choice followed if needed by those suggested by test selection in the computer program. Thus the number and nature of these tests varied from strain to strain; the average number was 29. The success obtained on the limited set of tests is approximately the same as that obtained on all the tests because the test selection process continued until all the tests with $a \geqslant 0.85$ and $\leqslant 0.15$ value between taxa pairs had been used or the strain had identified. This indicates, as expected, that tests with probabilities between 0.8 and 0.2 play little part in discriminating between taxa pairs, and that identi- 
fication by our method can save tests without losing identification accuracy. Tables 4 and 5 also show that, when the results can be compared, the identification rates on Matrix 66 were inferior to those obtained on Matrix 68.

The assessment of success is complex and all the cases are shown separately in Tables 4 and 5. When both computer and conventional identification place the strain in the same taxon, success can be assumed. When both computer and conventional identification fail to identify the strain it can be counted as an agreement, but represents the percentage of strains in a sample which cannot be identified by either means. When the computer identification of a strain indicates a different taxon from that of the conventional identification, then either might have been correct unless additional evidence, e.g. serological, was available. This dilemma was most frequent with the less satisfactory Matrix 66 and more common with field strains than reference strains as might be expected. In order to interpret our results as stringently as possible we have considered this situation to represent a failure of computer identification. When the computer identified a strain which could not be identified by conventional means, we have likewise not included it in the success rate and have shown the percentages separately in Tables 4 and 5 , although in fact the computer identification might be correct.

Table 4 shows that an identification rate of $90.8 \%$ was achieved on Matrix 68 for reference strains of fermentative bacteria, and Table 5 that a corresponding rate of only $82 \cdot \mathrm{I} \%$ was reached for non-fermentative bacteria on this matrix. The marked increased rate of success (nearly $40 \%$ ) for non-fermentative bacteria by Matrix 68 compared to Matrix 66 is due to improvements in the data after standardized tests on strains.

With field strains on Matrix 68, computer and conventional identification methods agreed in $89.4 \%$ of fermentative strains and $70.8 \%$ of non-fermentative strains. Failure to identify by both means is counted as an agreement. The series of strains tested on Matrix 66 are not comparable with those on Matrix 68 since the latter contained a large number of strains, collected for epidemiological purposes, for which the matrix is not suitable such as strains of Pseudomonas spp. not included in the matrices.

Most of the field strains in these series had caused difficulty in identification in the medical diagnostic bacteriological laboratory and many strains were atypical. Similarly the series of reference strains contained many unusual strains. In general, greater success rates could be expected with typical strains, and in fact have been found with the typical field strains which we received.

Taxa not in the matrix. Apart from some contaminants and Gram-positive organisms received in error which did not identify, 5I strains were tested which belonged to taxa conforming to the original definition but not in the matrix. These strains were chosen because they were closely related to taxa in the matrix or likely to cause confusion. On Matrix 68, II of these $5 \mathrm{I}$ strains $(2 \mathrm{I} .6 \%)$ identified in a taxon in the matrix (Bascomb et al. 1973). However, these strains either did not differ in their test results from any matrix entries of 0.99 or 0.0 I for the taxon into which they were identified or at most in one or two tests (Bascomb et al. 1973). Clearly further tests able to discriminate between the taxa concerned are required for the matrix. The method adopted by which the computer printout indicated those tests where the taxon into which the strain identified had a value of 0.99 or 0.0 I and the strain had given a contradictory negative or positive result served as a warning in many cases that the strain might belong to a taxon not in the matrix.

Test differences, frequency of usage, separation value. In Table $\mathrm{I}$ the tests are arranged by the frequency with which they were used by the sending laboratories in their initial set. Also included are the separation values of the tests and the interlaboratory test differences. 
Table 6. Separation values of 49 tests for fermentative taxa

$\begin{array}{ccc} & \begin{array}{c}\text { Separation } \\ \text { value }(S)\end{array} & S \text { as a } \\ \text { percentage } \\ \text { Test } & (\max .=784) & \text { of maximum }\end{array}$

Arabinose, acid production

Motility at RT

Rhamnose, acid production

ONPG ( $\beta$-galactosidase)

Gas production from glucose

Sorbitol, acid production

Ornithine, decarboxylase

$\mathrm{KCN}$ medium growth

Indole production

Lysine, decarboxylase

Motility at $37^{\circ} \mathrm{C}$

Sucrose, acid production

Methyl red at RT

Arginine, dihydrolase

Raffinose, acid production

Gelatin liquefaction within 28 days

Xylose, acid production

Inositol, acid production

$\mathrm{H}_{2} \mathrm{~S}$ production (TSI)

Lactose, acid production

Malonate utilization

Mannitol, acid production

Simmons' citrate, growth

Methyl red at $37^{\circ} \mathrm{C}$

Salicin, acid production

$\begin{array}{ll}726 & 93 \\ 700 & 89 \\ 696 & 89 \\ 660 & 84 \\ 648 & 83 \\ 625 & 80 \\ 624 & 80 \\ 616 & 79 \\ 595 & 76 \\ 580 & 74 \\ 572 & 73 \\ 546 & 70 \\ 544 & 69 \\ 544 & 69 \\ 532 & 68 \\ 525 & 67 \\ & \\ 504 & 64 \\ 480 & 61 \\ 473 & 60 \\ 455 & 58 \\ 451 & 57 \\ 450 & 57 \\ 448 & 57 \\ 442 & 56 \\ 435 & 55 \\ & \end{array}$

* All taxa are fermentative.

$\mathrm{RT}$, room temperature or incubator at 22 or $30^{\circ} \mathrm{C}$.

$$
\begin{array}{cc}
\text { Separation } & S \text { as a } \\
\text { value }(S) & \text { percentage } \\
\text { (max. }=784) & \text { of maxim um }
\end{array}
$$

Gelatin liquefaction within

414

53

$\mathrm{H}_{2} \mathrm{~S}$ production (lead acetate paper)

Cellobiose, acid production

Oxidase, presence

Adonitol, acid production

Gluconate reduction

Maltose, acid production

Trehalose, acid production

Urease, presence

Glycerol, acid production

Voges-Proskauer at RT

Dulcitol, acid production

Deamination of phenyl-

alanine (PPA)

Selenite reduction

Starch, acid production

Growth at RT

Voges-Proskauer at $37^{\circ} \mathrm{C}$

Pigment production

MacConkey agar, growth

Growth at $37^{\circ} \mathrm{C}$

Catalase, presence

Hugh and Leifson test*

Nitrate reduction

Glucose, acid production

$\begin{array}{rr}396 & 5 \mathrm{I} \\ 390 & 50 \\ 378 & 48 \\ 378 & 48 \\ 360 & 46 \\ 352 & 45 \\ 352 & 45 \\ 342 & 44 \\ 330 & 42 \\ 308 & 39 \\ 30 \mathrm{I} & 38 \\ 300 & 38 \\ & \\ 279 & 36 \\ 252 & 32 \\ 144 & \mathrm{I} 8 \\ 138 & \mathrm{I} 8 \\ 109 & 14 \\ 88 & 1 \mathrm{I} \\ 55 & 7 \\ 0 & 0 \\ 0 & 0 \\ 0 & 0 \\ 0 & 0\end{array}$

TSI, triple sugar iron medium.

Discrete separation values are given for the three characteristics tested by the oxidation/ fermentation test (Hugh \& Leifson, 1953) but only a single figure for its frequency of usage as the three characteristics are tested together, and also a composite value for the test reproducibility since the test readings from which each characteristic is scored may affect the scoring of the other characteristics.

The frequency with which tests are chosen bears little relation to their separation value between the taxa or their interlaboratory reproducibility, nor does the frequency closely correspond to the date of introduction of the tests into bacteriology.

The separation value and order of rank of the tests in discrimination between the taxa depends on the number and nature of the taxa included. The separation values for the I4 non-fermentative and 56 fermentative taxa in Matrix 68 have been calculated separately (Tables 6 and 7) and show the differences in the separation values and in the ranking of the tests. The separation value for the Hugh and Leifson test has been calculated on an additive basis derived from the four alternative results.

These separation values reflect the poor discrimination of many of these tests between the taxa of non-fermentative bacteria. If the range of non-fermentative bacteria is extended, some of the tests with a separation score of o might become of value. Some tests although useful in discrimination with a particular result, e.g. pigment production, have a separation value of o since no included taxon had a probability over $85 \%$ for a positive result in that test. Similarly other subsets of taxa might be expected to show different separation values and rank. 


\section{Table 7. Separation values of 49 tests for non-fermentative taxa}

\begin{tabular}{|c|c|c|c|c|c|}
\hline Test & $\begin{array}{c}\text { Separation } \\
\text { value }(S) \\
(\max .=49)\end{array}$ & $\begin{array}{c}S \text { as a } \\
\text { percentage } \\
\text { of maximum }\end{array}$ & Test & $\begin{array}{c}\text { Separation } \\
\text { value }(S) \\
(\max .=49)\end{array}$ & $\begin{array}{c}S \text { as a } \\
\text { percentage } \\
\text { of maximum }\end{array}$ \\
\hline Hugh and Leifson test* & 46 & 94 & Deamination of phenyl- & 0 & 0 \\
\hline Motility at RT & 42 & 86 & alanine (PPA) & & \\
\hline Arginine, dihydrolase & 36 & 73 & Indole production & 0 & 0 \\
\hline Motility at $37^{\circ} \mathrm{C}$ & 35 & $7 I$ & $\mathrm{H}_{2} \mathrm{~S}$ production (TSI) & 0 & 0 \\
\hline Oxidase, presence & 28 & 57 & $\mathrm{H}_{2} \mathrm{~S}$ production (lead & 0 & 0 \\
\hline Gelatin liquefaction within & 28 & 57 & acetate paper) & & \\
\hline 5 days & & & Lysine, decarboxylase & 0 & 0 \\
\hline Simmons' citrate, growth & 28 & 57 & Ornithine, decarboxylase & o & 0 \\
\hline Nitrate reduction & 24 & 49 & Methyl red at $37^{\circ} \mathrm{C}$ & 0 & 0 \\
\hline KCN medium, growth & $2 \mathrm{I}$ & 43 & Methyl red at RT & 0 & 0 \\
\hline MacConkey agar, growth & 20 & $4 I$ & Voges-Proskauer at $37^{\circ} \mathrm{C}$ & 0 & 0 \\
\hline Malonate utilization & 18 & 37 & Voges-Proskauer at RT & 0 & 0 \\
\hline Urease, presence & 18 & 37 & Gas production from glucose & 0 & o \\
\hline Glucose, acid production & 18 & 37 & Adonitol, acid production & 0 & 0 \\
\hline Gelatin liquefaction within & 15 & $3 \mathrm{I}$ & Cellobiose, acid production & 0 & 0 \\
\hline 28 days & & & Dulcitol, acid production & 0 & 0 \\
\hline Glycerol, acid production & 13 & 27 & Inositol, acid production & 0 & 0 \\
\hline Maltose, acid production & 13 & 27 & Lactose, acid production & 0 & 0 \\
\hline Growth at $37^{\circ} \mathrm{C}$ & 12 & 25 & Mannitol, acid production & 0 & 0 \\
\hline Gluconate reduction & 12 & 25 & Raffinose, acid production & 0 & 0 \\
\hline Selenite reduction & 12 & 25 & Rhamnose, acid production & 0 & 0 \\
\hline Arabinose, acid production & 12 & 25 & Salicin, acid production & 0 & 0 \\
\hline ONPG ( $\beta$-galactosidase) & 12 & 25 & Sorbitol, acid production & 0 & 0 \\
\hline Xylose, acid production & 12 & 25 & Starch, acid production & 0 & 0 \\
\hline Growth at RT & II & 22 & Sucrose, acid production & 0 & 0 \\
\hline \multirow[t]{2}{*}{ Catalase, presence } & 0 & 0 & Trehalose, acid production & 0 & 0 \\
\hline & & & Pigment production & 0 & 0 \\
\hline
\end{tabular}

* Discrimination between oxidative, alkaline and negative results.

$\mathrm{RT}$, room temperature or incubator at 22 or $30^{\circ} \mathrm{C}$. TSI, triple sugar iron medium.

An average test result difference of $7.9 \%$ was found between the sending laboratories and ours, derived from 452 field strains available for comparison in 13029 tests. This value is very similar to that of $6.9 \%$ found by Lapage et al. (1970) for 8002 tests. The test differences ranged from $30 \%$ for hydrogen sulphide production (tested by lead acetate papers over nutrient broth) to $2 \%$ for a number of tests. The values for individual tests are very similar to those found by Lapage et al. (1970). The results suggest that $\mathrm{H}_{2} \mathrm{~S}$ production, growth in Simmons's citrate and KCN media, nitrate reduction, acid production from starch, arabinose and sorbitol, late gelatin liquefaction, presence of urease and the methyl red and Voges-Proskauer tests at $37^{\circ} \mathrm{C}$ should be used with caution in one laboratory and are not reliable for overall interlaboratory comparisons. Several of these tests are widely known to give poor reproducibility, sometimes because the media and methods used differ or because they are difficult to read and interpret.

\section{DISCUSSION}

Taxa. Although the study was restricted to medically important taxa, ideally all bacteria which might be encountered should be represented in the matrix but this proved impracticable.

The taxa should be mutually exclusive, which is required for the use of conditional probabilities. Intermediate strains, common in some bacterial groups, e.g. enterobacteria, present a special problem. The characteristics within a taxon of bacteria are often variable, although 
some may be considered nearly constant for all members of the taxon. It is thus possible that a strain may have characteristics in common with two or more taxa and such an intermediate may be difficult to identify. For identification, prior classification is needed but a classification allowing many intermediate strains defeats its purpose while on the other hand, if the taxa in the classification are drawn widely to include most intermediate strains, then they may possess too few definite characteristics for successful identification. Compromises may have to be made in the construction of a matrix for probabilistic identification.

A numerical classification of strains based only on their results in the identification matrix could help to define taxa suitable for use in such a matrix. Reference strains identified by additional characteristics, should be included in such a classification to relate the results to more general classifications based on wider sets of tests.

On theoretical grounds, the taxa in a probability matrix should be of approximately equal rank since taxa of markedly higher rank could be divided into several taxa, unless such subdivisions prevented the subtaxa reaching an identification level as we found with the species of Shigella. In some cases, taxa were combined because the tests in the matrix did not separate them adequately, e.g. Moraxella spp.

Tests. The average difference of $7 \cdot 9 \%$ between the test results obtained in the sending laboratories and in ours made surprisingly little difference to the identification of the field strains as, on the sender's results, $79.6 \%$ of fermentative bacteria were identified compared to $8 \mathrm{I} \cdot 9 \%$ on the results of all the tests in our laboratory. Snell \& Lapage (I973) have found that degrees of variability may be more marked with particular strains or species and suggested that they might even be treated as characteristic of some taxa.

Poorly reproducible tests are still in wide use (Table I), probably by tradition although they may be more reproducible in one laboratory and may differentiate well between particular taxa in a limited situation. Overall differentiating power also plays only a small part in test choice (Table I). However, test choice is modified by the number of groups considered, by clinical information and by the likely bacteria to be found in the specimen under examination, e.g. faeces or sputum. Tests may also be chosen to select or exclude important pathogens without reference to their overall value, e.g. $\mathrm{H}_{2} \mathrm{~S}$ production by Salmonella. Additionally, the human mind judges more easily by tests in which only a few of the taxa give one type of result while the computer can make full use of the discriminating power of tests with a high separation value in which the number of positive and negative taxa are about equally divided; and the power of such tests becomes evident when they are used as a series.

By our method of test selection in the computer we were able, for 70 available additional strains, to complete the identification with, on average, 32 tests out of the $5 \mathrm{I}$ tests available; a figure similar to that found for 279 strains by Lapage et al. (1970), who achieved the same identification rate with an average of 29 tests as with 50 tests. In both cases, failure to identify both by computer and conventional means was considered as an 'identification'. In the present program test selection is not used until the initial set of tests have been carried out and further test economy might be achieved if test selection was used after the performance of a few tests of high separation value, perhaps in the future linked to an automated system.

Geographical variation. The taxa used for identification should correspond to those found in the particular population under sample. Two types of geographical variation should be distinguished, variation in the frequency of incidence of strains of the taxa and variation in the results of tests in a particular taxon, e.g. biotypes.

In the present study each taxon was treated as equally likely. When enough data has been collected to prepare frequency tables, these could be supplied to sending laboratories and changes with time of the frequency of the different taxa indicated. Alternatively two identi- 
fication scores could be calculated, one derived from the test results alone, and one from this score weighted by the relative frequencies.

The problems raised by sampling for the incidence of different biotypes in order to allot figures to the matrix are considerable. Apart from geographical variation discussed above, the uncertainty in the estimation of a rare event in the upper and lower limits (0.99 and 0.01) would require very large samples to reduce them. The data error method sets limits to the size of the samples.

However, the probability of biological prediction, i.e. the confidence which can be placed on predictions based on results derived from a given sample of living organisms about the future findings with subsequent strains of the same taxon, would seem to have narrower limits than that of the usual statistical estimates. The bacteria concerned have limited genetic possibilities so all the possible random distributions of characteristics are not available, e.g. if five strains of a new species of Pseudomonas were isolated and all were found to be Gramnegative then it would be unlikely both from this finding, and from previous experience of other strains of Pseudomonas that any further strains of the species would be Grampositive, though statisically one is only $95 \%$ confident that $48 \%$ or more strains of that species were Gram-negative. Such constant characters are frequently included in the definition of the taxon concerned and recognition of a subtaxon with a contradictory result may require redefinition of the taxon, e.g. the inclusion of the non-motile Pseudomonas mallei in the motile genus $P$ seudomonas. However, not all strains of a given taxon can be considered to have the properties given in the definition, e.g. although Enterobacteriaceae by definition produce catalase, strains of Shigella dysenteriae do not do so.

Uncompleted squares and independence of tests. The methods for unavailable information and inapplicable tests proved successful in our system but would be impracticable if the matrix contained many uncompleted squares as it would be difficult for a strain to reach identification level on the lowest possible score in a taxon with many uncompleted squares.

Scoring of uncompleted squares as 'no comparison' was tested, but proved unsatisfactory as the number of 'no comparisons' varied in each taxon so the identification scores were not necessarily derived from the same number of tests and no satisfactory method was found to compare these unequal products.

An uncompleted square can be allotted a probability of 0.5 which does not affect the identification but we did not use this method because in fact the value was unknown, and the results of the identification may prove misleading, which may be shown a posteriori when the true value is known.

Tests were causally linked in some taxa but a given test may be linked only in some taxa and not in others, also there is not enough knowledge of bacterial metabolism to evaluate the extent of linkage of test results among the taxa. Therefore in the present study the tests were treated as independent except those linked by logical definition or by performance of the test at different temperatures as explained before and in Willcox et al. (1973).

Identification rates. By normalizing likelihoods and fixing the identification level to 0.999 it was possible to predict what type of matrix would yield the best results. Firstly, in our system, each taxon would ideally differ from all others by at least two test probabilities, otherwise some of the strains of either taxon may not reach identification level in the present matrix, e.g. Escherichia coli and the Alkalescens-Dispar group. Secondly taxa containing 0.99 and 0.0 I probabilities are more likely to identify than those which contain intermediate values. However, if this is difficult to achieve, as in the case of a combined Shigella taxon, it might be practicable to lower the identification level for this group for strains for which it was necessary. 
The poor rates of identification of non-fermentative compared with fermentative strains (Tables 4,5 ) was largely due to the unsuitability of many of the tests, historically devised for fermentative bacteria, for differentiation between non-fermentative taxa. In addition, the inadequate classification of the bacterial taxa concerned may be reflected in a poor identification rate (Lapage, 1970), and the classification is poor of many non-fermentative bacteria. The rate of identification of Klebsiella strains was increased from $89 \%$ to $95 \%$ by the use of clusters formed in a numerical taxonomic study of strains of this group (Bascomb et al. 197I). A matrix is in preparation for non-fermentative bacteria with the addition of suitable tests, e.g. hydrolysis of tyrosine and of Tween 80 , and elimination of unsuitable ones, e.g. acid production in peptone water from a wide range of carbohydrates.

General. Computer and conventional identifications agreed in $90.8 \%$ of fermentative reference strains and $89.4 \%$ of fermentative field strains (Table 4 ) which shows the potential success of computer identification especially since many of the reference strains were unusual and the field strains had caused difficulty in identification. The method could well be extended to other groups of bacteria, with a library of matrices held in an identification centre. Our use of an on-line teleprinter and an interactive computer system has proved very successful. The poor rate of test reproducibility, the fact that a great deal of time is involved in correspondence about test differences and the number of occasions $(28 \%)$ on which the selected tests were not carried out by the sending laboratories (sometimes because these tests were not usually available in their laboratory) suggest that it might be preferable to carry out all the tests in the identification laboratory.

In conclusion, apart from research aspects into the use of conditional probability methods and the possible use of the system in other applications, the value of the study to the identification and the co-operating laboratories were: identification processes were clarified and made reproducible; the benefits of standardization of test records due to data processing were apparent and need to be extended to standardization of test methods; the availability by storage in the computer of large amounts of data for identification and test selection was proved successful; economy in tests was exercised by test strategy and a corresponding saving of time, labour and money; computer output enabled reports and laboratory data to be detailed, accessible and cumulative; the process was educative as co-operating laboratories introduced new tests suggested by test selection and became familiar with the characteristics of the rarer taxa, and a certain amount of modification of test methods occurred. Since the test results on the strains are stored in the computer, periodic review by numerical classification is made easy. Recognition of new taxa is aided and the more centralized a service the larger the number of strains available for the recognition of rarer clusters. The coding of clinical and similar data would provide opportunities for epidemiological and other clinical studies, and the potential of the system by linkage to automated apparatus must await the development, for microbiological assay, of automatic techniques as yet in their infancy.

We wish to thank Dr L. C. Payne, Dr W. Dybowski, and Mr D. A. Franklin, who in I963 first introduced the practical possibility of computer identification to the Central Public Health Laboratory, Colindale, and to the members of the Computer Subcommittee at this Institute, and particularly its chairman Dr S. T. Cowan who pioneered the project in its early stages. The first author wishes to pay tribute to the part played by Dr K. J. Steel in the original thought on the project and the construction of the first matrix. Our thanks are due to the Public Health Laboratory Service for help and support, and to the Department of Health and Social Security for the grant which made the work possible. 


\section{REFERENCES}

BAER, H. \& WASHINGTON, L. (1972). Numerical diagnostic key for the identification of Enterobacteriaceae. Applied Microbiology 23, 108-1 I 2.

Bascomb, S., Lapage, S. P., Curtis, M. A. \& Willcox, W. R. (I973). Identification of bacteria by computer: identification of reference strains. Journal of General Microbiology 77, 29I-3I 5.

Bascomb, S., Lapage, S. P., Willcox, W. R. \& CuRTis, M. A. (I97I). Numerical classification of the tribe Klebsielleae. Journal of General Microbiology 66, 279-295.

BeERS, R. J. \& LOCKHART, W. R. (1962). Experimental methods in computer taxonomy. Journal of General Microbiology 28, 633-640.

Dito, W. R. (1971). Personal communication of desk-top computer applications in the clinical laboratory, presented as Application of Programmable Calculators to Microbiology at Commission Continuing Education Workshops, American Society Clinical Pathology, Spring and Fall meetings 1970 and 197 I.

Dybowski, W. \& Franklin, D. A. (1968). Conditional probability and the identification of bacteria: a pilot study. Journal of General Microbiology 54, 2 I 5-229.

FEY, H. (1959). Differenzierungsschema für gramnegative aerobe Stäbchen. Schweizerische Zeitschrift für Allgemeine Pathologie und Bakteriologie 22, 64I-652.

Gyllenberg, H. (1963). A general method for deriving determination schemes for random collections of microbial isolates. Annales Academiae scientiarum fennicae A, IV 69, I-23.

GyllenberG, H. G. (1965). A model for computer identification of microorganisms. Journal of General Microbiology 39, 40I-405.

HILl, L. R. \& SilveSTRI, L. G. (I962). Quantitative methods in the systematics of Actinomycetales. III. The taxonomic significance of physiological-biochemical characters and the construction of a diagnostic key. Giornale di Microbiologia 10, $\mathrm{I}-28$.

Hugh, R. \& LEIFSON, E. (1953). The taxonomic significance of fermentative versus oxidative metabolism of carbohydrates by various Gram-negative bacteria. Journal of Bacteriology 66, 24-26.

LAPAGE, S. P. (1970). Identification services in the National Collection of Type Cultures. In Proceedings of the First International Conference of Culture Collections, pp. I7I-18I. Edited by H. Iizuka and T. Hasegawa. Tokyo: University of Tokyo Press.

Lapage, S. P., Bascomb, S., Willcox, W. R. \& Curtis, M. A. (1970). Computer identification of bacteria. In Automation, Mechanization and Data Handling in Microbiology, pp. I-22. Edited by A. Baillie and R. J. Gilbert. Society for Applied Bacteriology Technical Series no. 4. London: Academic Press.

Maccacaro, G. A. (1958). La misura della informazione contenuta nei criteri di classificazione. Annali di Microbiologia ed Enzimologia 8, 23 I-239.

MöllER, F. (1962). Quantitative methods in the systematics of Actinomycetales. IV. The theory and application of a probabilistic identification key. Giornale di Microbiologia ro, 29-47.

Niemelä, S. I., Hopkins, J. W. \& Quadling, C. (1968). Selecting an economical binary test battery for a set of microbial cultures. Canadian Journal of Microbiology 14, 27 I-279.

PAYNe, L. C. (1963). Towards medical automation. World Medical Electronics 2, 6-I I.

Quadling, C. \& Colwell, R. R. (I964). The use of numerical methods in characterizing unknown isolates. Developments in Industrial Microbiology 5, $15 \mathrm{I}-\mathrm{I} 6 \mathrm{I}$.

RYPKA, E. W. \& BABB, R. (1970). Automatic construction and use of an identification scheme. Medical Research Engineering 9, 9-19.

Rypka, E. W., Clapper, W. E., Bowen, I. G. \& BabB, R. (1967). A model for the identification of bacteria. Journal of General Microbiology 46, 407-424.

SNeAth, P. H. A. (1969). Computers in bacteriology. Journal of Clinical Pathology 22 (suppl.) (College of Pathologists) 3, 87-92.

SNeath, P. H. A. \& Johnson, R. (1972). The influence on numerical taxonomic similarities of errors in microbiological tests. Journal of General Microbiology 72, 377-392.

SNELl, J. J. S. \& LAPAGE, S. P. (I973). Carbon source utilization tests as an aid to the classification of nonfermenting bacteria. Journal of General Microbiology 74, 9-20.

STEEL, K. J. (I962). The practice of bacterial identification. In Microbial Classification: Twelfth Symposium of the Society for General Microbiology, pp. 405-432. Edited by G. C. Ainsworth and P. H. A. Sneath. Cambridge University Press.

STEEL, K. J. (1965). Microbial identification. Journal of General Microbiology 40, I43-I48.

Willcox, W. R., Lapage, S. P., Bascomb, S. \& CuRtis, M. A. (1973). Identification of bacteria by computer: theory and programming. Journal of General Microbiology 77, 317-330. 